\title{
Algebraic Methods for Computer Aided Geometric Design
}

Thomas W. Sederberg

tom@cs.byu.edu

Jianmin Zheng

Follow this and additional works at: https://scholarsarchive.byu.edu/facpub

Part of the Computer Sciences Commons

\section{Original Publication Citation}

T. W. Sederberg and J. Zheng, "Algebraic methods for computer aided geometric design," Chapter 15 in Handbook of Computer Aided Geometric Design, G. Farn, J. Hoschek, and M.-S. Kim, eds., Elsevier, 22.

\section{BYU ScholarsArchive Citation}

Sederberg, Thomas W. and Zheng, Jianmin, "Algebraic Methods for Computer Aided Geometric Design" (2002). Faculty Publications. 5.

https://scholarsarchive.byu.edu/facpub/5

This Book Chapter is brought to you for free and open access by BYU ScholarsArchive. It has been accepted for inclusion in Faculty Publications by an authorized administrator of BYU ScholarsArchive. For more information, please contact ellen_amatangelo@byu.edu. 


\section{Algebraic Methods for Computer Aided Geometric Design}

Thomas W. Sederberga and Jianmin Zheng $^{\mathrm{b}}$

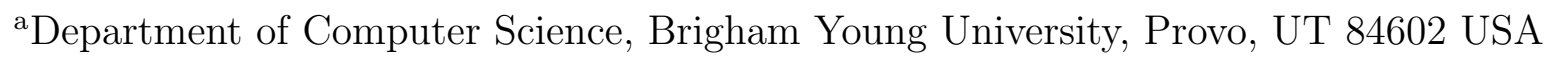

${ }^{\mathrm{b}}$ Department of Mathematics, Zhejiang University, Hangzhou, Zhejiang, China

Silhouettes (polar curves), blend curves, inversion, implicitization, offset curves, singularities,

\section{Introduction}

CAGD draws from several branches of mathematics and computer science, such as approximation theory, differential geometry, and numerical analysis. This chapter reviews some of the tools of algebra and algebraic geometry that have been brought to bear on problems in CAGD.

Most of the free-form curves and surfaces used in CAGD are given by parametric equations. Planar curves in CAGD are typically defined as

$x=\frac{a(t)}{c(t)}, \quad y=\frac{b(t)}{c(t)}$

where $a(t), b(t)$, and $c(t)$ are polynomials in the Bernstein basis for rational Bézier curves or in the B-spline basis for NURBS. Algebraic methods most commonly use polynomials in the power basis: $a(t)=a_{0}+a_{1} t+\cdots+a_{n} t^{n}$, etc. Polynomials can be converted from Bernstein basis to power basis, although some algebraic methods such as resultants can be formulated using the Bernstein basis.

Parametric surfaces in CAGD are defined

$x=\frac{a(s, t)}{d(s, t)}, \quad y=\frac{b(s, t)}{d(s, t)}, \quad z=\frac{c(s, t)}{d(s, t)}$

where $a(s, t), b(s, t), c(s, t)$ and $d(s, t)$ are polynomials.

Surfaces and plane curves can also be defined using implicit equations. One contribution that algebraic methods make to CAGD is in solving the problem of implicitization and inversion of parametric curves and surfaces. For any parametric curve given by (1), an implicit equation $f(x, y)=0$ (where $f(x, y)$ is a polynomial) exists that desribes exactly the same curve. Likewise, for any paramtric surface given by (2), there exists an implicit equation $f(x, y, z)=0$ that describes exactly the same surface. The process of finding the implicit equation of a parametric curve or surface is called implicitization. Implicitization is of value in CAGD because the problem of determining whether a given point lies on a curve or surface is addressed much more easily using the implicit form than the parametric form. Implicitization is discussed in Section ??. 
An inversion formula for a parametric curve (1) is of the form $t=\frac{g(x, y)}{h(x, y)}$ where $g$ and $h$ are polynomials. An inversion formula returns the parameter value $t$ corresponding to a point $(x, y)$ that lies on the curve. Inversion is discussed in Section ??.

Algebraic methods also can facilitate the design of algorithms for computing intersections between curves and surfaces. The intersection problem is surveyed in Section ??. Some additional issues, such as how to detect singularities and how to compute silhouette points, are overviewed in Section ??.

Many of the algebraic methods reviewed in this chapter come from classical analytic geometry. The twentieth century witnessed a marked shift from the constructive approach to non-constructive.

\subsection{Notation and terminology}

In general, a polynomial in $n$ variables $x_{1}, \ldots, x_{n}$ is defined

$f\left(x_{1}, x_{2}, \ldots, x_{n}\right)=\sum_{i=1}^{\tau} c_{i} x_{1}^{e_{1, i}} x_{2}^{e_{2, i}} \cdots x_{n}^{e_{n, i}}$.

Each summand $c_{i} x_{1}^{e_{1, i}} x_{2}^{e_{2, i}} \cdots x_{n}^{e_{n, i}}$ is called a term, $x_{1}^{e_{1, i}} x_{2}^{e_{2, i}} \cdots x_{n}^{e_{n, i}}$ is a monomial, and $c_{i}$ is the coefficient of the monomial. By convention, any given monomial occurs in at most one term in a polynomial.

$k\left[x_{1}, \ldots, x_{n}\right]$ signifies the set of all polynomials in the variables $x_{1}, \ldots, x_{n}$ whose coefficients belong to a field $k$. For example, $R[x, y]$ is the set of all polynomials

$\sum c_{i} x^{e_{1, i}} y^{e_{2, i}}$

where $c_{i} \in R$ and $e_{1, i}, e_{2, i} \in\{0,1,2, \ldots\}$. Thus, " $f \in R[x, y, z]$ " means that $f$ is a polynomial whose variables are $x, y$ and $z$ and whose coefficients are real numbers. All polynomials in this chapter have coefficients that are real numbers.

It is often useful to list the terms of a polynomial in decreasing order, beginning with the leading term. This is done using a term order - a way to compare any two distinct terms of a polynomial and declare which is "greater."

For linear polynomials, term order amounts to merely declaring an order on the variables. For example, the terms of the polynomial

$2 x+3 y-4 z$

are in proper order if we declare $x>y>z$. If we declare $y>z>x$, the proper order would be $3 y-4 z+2 x$. For non-linear polynomials, we begin by declaring an order on the variables, and then we must also choose one of several schemes that decide how the exponents in a polynomial influence term order. One such scheme is called lexicographical order (nicknamed lex), defined as follows. If the variables of a polynomial are ordered $x_{1}>x_{2}>\ldots>x_{n}$, then given two distinct terms $T_{i}=c_{i} x_{1}^{e_{1, i}} x_{2}^{e_{2, i}} \cdots x_{n}^{e_{n, i}}$ and $T_{j} c_{j} x_{1}^{e_{1, j}} x_{2}^{e_{2, j}} \cdots x_{n}^{e_{n, j}}, T_{i}>T_{j}$ if

1. $e_{1, i}>e_{1, j}$, or if

2. $e_{1, i}=e_{1, j}$ and $e_{2, i}>e_{2, j}$, or, in general, if

3. $e_{k, i}=e_{k, j}$ for $k=1, \ldots, m-1$ and $e_{m, i}>e_{m, j}$. 
For example, the polynomial

$3 x^{2} y^{2} z+4 x y^{3} z^{2}+5 x^{3} z+6 y^{2}+7 x z^{3}+8$

using lex with $x>y>z$ would be written $5 x^{3} z+3 x^{2} y^{2} z+4 x y^{3} z^{2}+7 x z^{3}+6 y^{2}+8$ and its leading term is $5 x^{3} z$. Using lex with $z>x>y$ it would be written $7 z^{3} x+4 z^{2} x y^{3}+$ $5 z x^{3}+3 z x^{2} y^{2}+6 y^{2}+8$ and the leading term would be $7 z^{3} x$. Or using lex with $y>z>x$ would be written $4 y^{3} z^{2} x+3 y^{2} z x^{2}+6 y^{2}+7 z^{3} x+5 z x^{3}+8$ and the leading term would be $4 y^{3} z^{2} x$.

Another choice for term order is the degree lexicographical order (abbreviated deglex). If the variables are ordered $x_{1}>x_{2}>\ldots>x_{n}$, then using deglex, $T_{i}>T_{j}$ if

1. $e_{1, i}+e_{2, i}+\ldots+e_{n, i}>e_{1, j}+e_{2, j}+\ldots+e_{n, j}$, or

2. $e_{1, i}+e_{2, i}+\ldots+e_{n, i}=e_{1, j}+e_{2, j}+\ldots+e_{n, j}$ and $T_{i}>T_{j}$ with respect to lex.

Using deglex with $x>y>z$, the terms of $3 x^{2} y^{2} z+4 x y^{3} z^{2}+5 x^{3} z+6 y^{2}+7 x z^{3}+8$ would be ordered $4 x y^{3} z^{2}+3 x^{2} y^{2} z+5 x^{3} z+7 x z^{3}+6 y^{2}+8$.

As obverved in the lex and deglex examples, term orders ignore the coefficient of a term, so a term order might more properly be called a monomial order.

Other term orders can also be defined, such as degree reverse lexicographical order. The precise requirements for any term order are discussed in reference [6], page 18.

The n-dimensional real affine space is denoted $R^{n}$ and is the set of $n$-tuples:

$$
R^{n}=\left(a_{1}, \ldots, a_{n}\right): a_{1}, \ldots, a_{k} \in R
$$

\section{Ideals and Varieties}

This section presents a brief overview of ideals and varieties and suggests some ways how these topics fit into CAGD. An excellent treatment of ideals and varieties and their application to CAGD can be found in [22].

The polynomial ideal generated by $f_{1}, \ldots, f_{s} \in k\left[x_{1}, \ldots, x_{n}\right]$, denoted $\left\langle f_{1}, \ldots, f_{s}\right\rangle$, is defined

$\left\langle f_{1}, \ldots, f_{s}\right\rangle=\left\{p_{1} f_{1}+\ldots+p_{s} f_{s}: p_{i} \in k\left[x_{1}, \ldots, x_{n}\right]\right\}$.

Consider a set of polynomials $f_{1}, f_{2}, \cdots, f_{s} \in k\left[x_{1}, \ldots, x_{n}\right]$. Let $\left(a_{1}, \ldots, a_{n}\right)$ be a point in $k^{n}$ satisfying $f_{i}\left(a_{1}, \ldots, a_{n}\right)=0, i=1, \ldots, s$. The set of all such points $\left(a_{1}, \ldots, a_{n}\right)$ is called the variety defined by $f_{1}, \ldots, f_{s}$, and is denoted by $V\left(f_{1}, \ldots, f_{s}\right)$ :

$V\left(f_{1}, \ldots, f_{s}\right)=\left\{\left(a_{1}, \ldots, a_{n}\right) \in k^{n} \mid f_{i}\left(a_{1}, \ldots, a_{n}\right)=0, i=0, \ldots, s\right\}$.

A variety defined by a single polynomial - called a hypersurface - is the most familiar instance of a variety. A hypersurface in $R^{2}$ is a planar curve defined using an implicit equation, and a hypersurface in $R^{3}$ is what is normally called an implicit surface in CAGD. For example, $V\left(x^{2}+y^{2}-1\right)$ is a circle defined in terms of the implicit equation $x^{2}+y^{2}-1=0$ and $V(2 x+4 y-z+1)$ is the plane whose implicit equation is $2 x+4 y-z+1=0$.

A variety $V\left(f_{1}, \ldots, f_{s}\right)$ defined by more than one polynomial $(s>1)$ is the intersection of the varieties $V\left(f_{1}\right) \ldots V\left(f_{s}\right)$. 


\subsection{Gröbner Bases}

It can be very useful to devise alternative generators for an ideal. Necessary and sufficient conditions for $\left\langle f_{1}, \ldots, f_{n}\right\rangle=\left\langle g_{1}, \ldots, g_{m}\right\rangle$ are $f_{1}, \ldots, f_{n} \in\left\{g_{1}, \ldots, g_{m}\right\}$ and $g_{1}, \ldots, g_{m} \in\left\{f_{1}, \ldots, f_{n}\right\}$.

A Gröbner basis of an ideal $I$ is a set of polynomials $\left\{g_{1}, \ldots, g_{t}\right\}$ such that the leading term of any polynomial in $I$ is divisible by the leading term of at least one of the polynomials $g_{1}, \ldots, g_{t}$. This, of course, requires that a term order be fixed for determining the leading terms: different term orders produce different Gröbner bases. Several excellent books have been written on Gröbner bases that do not presuppose that the reader has an advanced degree in mathematics $[22,6,13]$

A Gröbner basis is a particularly attractive set of generators for an ideal, as illustrated by two familiar examples. If $\left\{f_{1}, \ldots, f_{s}\right\}$ are polynomials in one variable, the Gröbner basis of $\left\langle f_{1}, \ldots, f_{n}\right\rangle$ consists of a single polynomial: the GCD of $f_{1}, \ldots, f_{s}$. If $\left\{f_{1}, \ldots, f_{s}\right\}$ are linear polynomials in several variables, the Gröbner basis is an uppertriangular form of a set of linear equations. The Gröbner basis of these special cases provides significant computational advantage and greater insight, and the same is true of the Gröbner basis of a more general ideal.

Gröbner bases are the fruit of Bruno Buchberger's Ph.D. thesis [15], and are named in honor of his thesis advisor. Buchberger devised an algorithm for computing Gröbner bases [16,22]. Also, commercial software packages such as Maple and Mathematica include capabilities for computing Gröbner bases.

\section{Resultants}

Given a set of polynomials in several variables, elimination theory studies criteria for the existence of common solutions, i.e., whether the variety of the ideal generated by these polynomials is nonempty. While Gröbner basis methods can be used to accomplish this task, resultants developed in classical algebra provide a powerful tool for this purpose [20, 21], and are usually much more efficient than Gröbner bases in practical applications.

Resultants are a set of integer polynomials in the coefficients of the given polynomials such that the vanishing of the resultants is the necessary and sufficient conditions for the given polynomials to have common solutions. They were developed based on the theory of determinant in the late 19 th century and the early 20 th century [40,55]. The main idea is to construct from the given set of polynomials, a possibly large system of independent polynomials such that they generate the same ideal as the original polynomial set and contain as many polynomials as terms in the polynomials so that each term can be used as an unknown and the theory of linear system of equations can be applied. In practice, the resultants for two univariate polynomials and for three bivariate polynomials are most of interest.

\subsection{Sylvester's resultant}

Consider the two polynomials

$$
\begin{cases}f(t)=a_{n} t^{n}+a_{n-1} t^{n-1}+\cdots+a_{1} t+a_{0}, & a_{n} \neq 0 \\ g(t)=b_{m} t^{m}+b_{m-1} t^{m-1}+\cdots+b_{1} t+b_{0}, & b_{m} \neq 0\end{cases}
$$


Using Sylvester's dialytic method, we multiply $f(t)$ by $t^{m-1}, t^{m-2}, \cdots, t, 1$ and $g(t)$ by $t^{n-1}, \cdots, t, 1$, arriving at $m+n$ polynomials, which can be arranged in matrix form:

$$
\left[\begin{array}{c}
t^{m-1} f(t) \\
\vdots \\
t f(t) \\
f(t) \\
t^{n-1} g(t) \\
\vdots \\
t g(t) \\
g(t)
\end{array}\right]=\left[\begin{array}{cccccccc}
a_{n} & a_{n-1} & \cdots & a_{1} & a_{0} & & & \\
& a_{n} & a_{n-1} & \cdots & a_{1} & a_{0} & & \\
& & \cdots & \cdots & & & & \\
& & & & a_{n} & \cdots & \cdots & a_{0} \\
b_{m} & b_{m-1} & \cdots & b_{1} & b_{0} & & & \\
& b_{m} & b_{m-1} & \cdots & b_{1} & b_{0} & & \\
& & \cdots & \cdots & & & & \\
& & & & b_{m} & \cdots & \cdots & b_{0}
\end{array}\right]\left[\begin{array}{c}
t^{m+n-1} \\
t^{m+n-2} \\
\cdot \\
\cdot \\
\cdot \\
\cdot \\
t \\
1
\end{array}\right]
$$

The ideal generated by these polynomials is just the ideal $\langle f(t), g(t)\rangle$, and thus has the same variety. The determinant of the above coefficient matrix, written $R(f, g)$, is an expression in terms of the coefficients $a_{i}$ and $b_{i}$. It is the well-known Sylvester's resultant for $f(t)$ and $g(t)$.

The Sylvester's resultant can also be obtained by Euler's method. Euler's method can easily show that $R(f, g)=0$ is not only the necessary but also sufficient condition for $f(t)$ and $g(t)$ to have common roots. In fact, we introduce two polynomials $h(t)$ of degree $m-1$ and $k(t)$ of degree $n-1$ with coefficients undetermined. Letting $h(t) f(t)-k(t) g(t)=0$ leads to $m+n$ linear equations with $m+n$ unknowns which are the coefficients of $h(t)$ and $k(t)$. The determinant of the coefficient matrix of the linear equations is just the Sylvester's resultant. Obviously the determinant vanishes if and only if there exist nonzero polynomials $h(t)$ and $k(t)$ such that $h(t) f(t)=k(t) g(t)$ holds. This is equivalent to the existence of the common roots of polynomials $f(t)$ and $g(t)$.

\subsection{Bezout's resultant}

Another popular resultant formulation for two univarite polynomials is Bezout's method. Bezout's resultant has more compact dimension than Sylvester's resultant. A nice derivation of Bezout's resultant is due to Cayley. Without loss of generality, we assume the degree of the polynomials $f(t)$ is greater than or equal to the degree of $g(t)$, i.e., $n \geq m$. Construct a symmetric function

$d(t, s)=\left|\begin{array}{ll}f(t) & f(s) \\ g(t) & g(s)\end{array}\right| /(s-t)=\frac{f(t) g(s)-f(s) g(t)}{s-t}$

Some algebraic calculations show

$$
\begin{aligned}
d(t, s) & =f(t)(g(t)-g(s)) /(t-s)-g(t)(f(t)-f(s)) /(t-s) \\
& =\sum_{k=0}^{m-1}\left(f \sum_{i=k+1}^{m} b_{i} t^{i-k-1}-g \sum_{i=k+1}^{n} a_{i} t^{i-k-1}\right) s^{k}-\sum_{k=m}^{n-1}\left(g \sum_{i=k+1}^{n} a_{i} t^{i-k-1}\right) s^{k}
\end{aligned}
$$

Thus $d(t, s)$ is an $n-1$ degree polynomial in $s$. The coefficients of $s^{k}, k=0, \ldots, n-1$, give $n$ polynomials of $t$ similar to those obtained from Sylvester's method. It is easy to know that the variety of the ideal generated by these polynomials is the same as $V(\langle f, g\rangle)$. 
Write these polynomials in matrix form:

$$
\left[\begin{array}{c}
f \sum_{i=1}^{m} b_{i} t^{i-1}-g \sum_{i=1}^{n} a_{i} t^{i-1} \\
\vdots \\
f b_{m}-g \sum_{i=m}^{n} a_{i} t^{i-m} \\
-g \sum_{i=m+1}^{n} a_{i} t^{i-m-1} \\
\vdots \\
-g a_{n}
\end{array}\right]=\left[\begin{array}{ccc}
c_{00} & \cdots & c_{0, n-1} \\
\vdots & \ddots & \vdots \\
c_{n-1,0} & \cdots & c_{n-1, n-1}
\end{array}\right]\left[\begin{array}{c}
1 \\
t \\
\vdots \\
t^{n-1}
\end{array}\right]
$$

with the entry $c_{i j}=\sum_{\substack{k \leq \min (i, j) \\ k+h=i+j+1}}\left(a_{k} b_{h}-a_{h} b_{k}\right)$ and the convention that $b_{m+1}=\cdots=b_{n}=0$.

This coefficient matrix is called Bezout's matrix. Thus, if $V(\langle f, g\rangle) \neq\{0\}$, the determinant of Bezout's matrix must vanish. The converse is also true when $n=m$, the proof of which can be found in $[26,29]$. The determinant is therefore a resultant for $f$ and $g$, known as Bezout's resultant.

When $n>m$, the Bezout's determinant will have an extraneous factor of $a_{n}^{n-m}$. This extraneous factor can be removed by modifying the Bezout's resultant as follows [24]: The first $m$ polynomials are the same as in (7); and the rest $n-m$ polynomials are obtained from $t^{i-m} g(t), i=m, \ldots, n$. Thus $c_{i j}=b_{i+j-m}$ for $i \geq m, 0 \leq j \leq n-1$. Look at the example of $f(t)=a_{2} t^{2}+a_{1} t+a_{0}$ and $g(t)=b_{1} t+b_{0}$. The previous Bezout's determinant is $\left|\begin{array}{cc}a_{0} b_{1}-a_{1} b_{0} & -a_{2} b_{0} \\ -a_{2} b_{0} & -a_{2} b_{1}\end{array}\right|=a_{2}\left|\begin{array}{cc}a_{0} b_{1}-a_{1} b_{0} & -a_{2} b_{0} \\ -b_{0} & -b_{1}\end{array}\right|$ and the modified Bezout's resultant is $\begin{array}{cc}a_{0} b_{1}-a_{1} b_{0} & -a_{2} b_{0} \\ b_{0} & b_{1}\end{array}$

\subsection{Dixon's resultant}

The Cayley's formulation can be extended to the three bivariate polynomial case. Consider three polynomials:

$$
f(s, t)=\sum_{i=0}^{n} \sum_{j=0}^{m} a_{i j} s^{i} t^{j}, \quad g(s, t)=\sum_{i=0}^{n} \sum_{j=0}^{m} b_{i j} s^{i} t^{j}, \quad h(s, t)=\sum_{i=0}^{n} \sum_{j=0}^{m} c_{i j} s^{i} t^{j}
$$

Dixon observed that the expression

$$
d(s, t, \alpha, \beta)=\left|\begin{array}{lll}
f(s, t) & g(s, t) & h(s, t) \\
f(s, \beta) & g(s, \beta) & h(s, \beta) \\
f(\alpha, \beta) & g(\alpha, \beta) & h(\alpha, \beta)
\end{array}\right| /(s-\alpha)(t-\beta)
$$

is actually a polynomial of degree $2 n-1, m-1, n-1$ and $2 m-1$ in $s, t, \alpha, \beta$ respectively. Thus it can be written as $d(s, t, \alpha, \beta)=\sum_{i j k l} s^{i} t^{j} \alpha^{k} \beta^{l}$ where

$$
d_{i j k l}=\sum_{\begin{array}{c}
u+u^{\prime}+u^{\prime \prime}=i \\
v+v^{\prime}+v^{\prime \prime}=l
\end{array}}\left|\begin{array}{lll}
a_{u v} & a_{u^{\prime}, v^{\prime}+j+1} & a_{u "+k+1, v^{\prime \prime}} \\
b_{u v} & b_{u^{\prime}, v^{\prime}+j+1} & b_{u "+k+1, v^{\prime \prime}} \\
c_{u v} & c_{u^{\prime}, v^{\prime}+j+1} & c_{u "+k+1, v^{\prime \prime}}
\end{array}\right|
$$




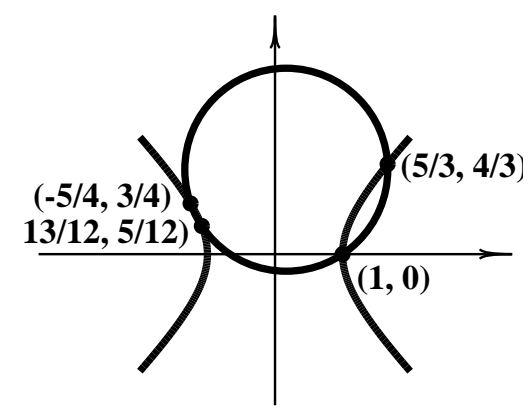

Figure 1. Circle and Hyperbola

For any $(s, t) \in V(\langle f, g, h\rangle), d(s, t, \alpha, \beta)$ vanishes no matter what $\alpha$ and $\beta$ are. Thus the coefficients of each $\alpha^{k} \beta^{l}$ must vanish at these $(s, t)$ pairs. This gives $2 m n$ polynomials in $s$ and $t$. The determinant of the coefficient matrix from these polynomials is a resultant for $f, g$ and $h$, called the Dixon's resultant [27].

\section{Intersection Computations}

The algebraic tools of Gröbner bases and resultant empower us to solve several problems of interest to CAGD. This section looks at several examples of intersection computations.

As noted in Section 2 , a variety $V\left(f_{1}, \ldots, f_{s}\right)$ defined by more than one polynomial $(s>$ 1 ) is the intersection of the varieties $V\left(f_{1}\right) \ldots V\left(f_{s}\right)$. Therefore, intersection computation is equivalent to determining a variety.

A common way to compute intersections is to us a multivariate Newton algorithm. A serious problem with this approach is robustness: it is difficult or impossible to assure that all intersection points have been found. Algebraic tools such as Gröber bases and resultants provide a systematic method for computing a variety, with the assurance that all intersection points have been found. The general strategy is based on the simple observation that

$V\left(f_{1}, \ldots, f_{s}\right)=V\left(\left\langle f_{1}, \ldots, f_{s}\right\rangle\right)$.

Consequently, if $f_{1}, \ldots, f_{s}$ and $g_{1}, \ldots, g_{t}$ are bases of the same ideal, then

$V\left(f_{1}, \ldots, f_{s}\right)=V\left(g_{1}, \ldots, g_{t}\right)$.

\subsection{Algebraic curve intersection}

Consider the example in Figure 1 of a hyperbola $V\left(x^{2}-y^{2}-1\right)$ and a circle $V\left(6 x^{2}+6 y^{2}-2 x-15 y-4\right)$. The set of points at which the circle and hyperbola intersect is the variety $V\left(6 x^{2}+6 y^{2}-2 x-15 y-4, x^{2}-y^{2}-1\right)=$ $\left\{\left(\frac{5}{3}, \frac{4}{3}\right),(1,0),\left(-\frac{13}{12},-\frac{5}{12}\right),\left(-\frac{5}{4}, \frac{3}{4}\right)\right\}$. Therefore, the problem of computing the intersection of two algebraic curves $f_{1}(x, y)=0$ and $f_{2}(x, y)=0$ amounts to computing the variety $V\left(f_{1}, f_{2}\right)$. 
is provided by Groöbner bases provide an algorithm for systematically solving for all points in $V\left(f_{1}, f_{2}\right)$, by virtue of the simple observation that

$V\left(f_{1}, \ldots, f_{s}\right)=V\left(\left\langle f_{1}, \ldots, f_{s}\right\rangle\right)$.

Consequently, if $f_{1}, \ldots, f_{s}$ and $g_{1}, \ldots, g_{t}$ are bases of the same ideal, then

$V\left(f_{1}, \ldots, f_{s}\right)=V\left(g_{1}, \ldots, g_{t}\right)$.

The practical value of this fact can be illustrated from the problem of computing the points at which the circle and hyperbola in Figure 1 intersect. It can be verified that

$\left\langle 6 x^{2}+6 y^{2}-2 x-15 y-4, x^{2}-y^{2}-1\right\rangle=$

$\left\langle-12 x^{2}+2 x+15 y+10,144 x^{4}-48 x^{3}-461 x^{2}+40 x+325\right\rangle \quad$ (the Gröbner basis)

Since any point of intersection must be zeros of all generators of the ideal, the only possible $\mathrm{x}$-coordinates for the intersection points must be roots of $144 x^{4}-48 x^{3}-461 x^{2}+40 x+$ $325=0$ (the roots are $\frac{5}{3}, 1,-\frac{13}{12}$, and $-\frac{5}{4}$ ). The corresponding $y$ coordinates can then be solved using $-12 x^{2}+2 x+15 y+10=0$.

\section{Implicitization and Inversion}

Any polynomial parametric curve or surface can be represented exactly using an implicit equation. For example, a circle can be expressed using the parametric equation $x=$ $2 t /\left(t^{2}+1\right), y=\left(t^{2}-1\right) /\left(t^{2}+1\right)$ or using the implicit equation $x^{2}+y^{2}-1=0$.

\begin{tabular}{|l|l|l|}
\hline & Parametric & Implicit \\
\hline Line & $x=2 t+1, y=t-2$ & $x-2 y-5=0$ \\
Circle & $x=2 t /\left(t^{2}+1\right), y=\left(t^{2}-1\right) /\left(t^{2}+1\right)$ & $x^{2}+y^{2}-1=0$ \\
Sphere & $x=\frac{2 t}{s^{2}+t^{2}+1}, y=\frac{2 s}{s^{2}+t^{2}+1}, z=\frac{s^{2}+t^{2}-1}{s^{2}+t^{2}+1}$ & $x^{2}+y^{2}+z^{2}-1=0$ \\
Steiner Surface & \\
\hline
\end{tabular}

\section{Parametric Curve Intersection}

\section{Silhouette points}

\section{PLANAR ALGEBRAIC CURVES}

\subsection{Genus and Parametrization}

We have seen that every parametric curve can be expressed in implicit form. The reverse is not generally true. The condition under which an implicit curve can be parametrized using rational polynomials is that its genus must be zero. Basically, the genus of a curve is given by the formula $g=\frac{(n-1)(n-2)}{2}-d$ where $g$ is the genus, $n$ is the degree, and $d$ is the number of double points. There are some subtleties involved in this equation, but we will not concern ourselves with them. They deal with more complicated multiple points.

We see immediately that all curves of degree one and two have genus zero and thus can be parametrized using rational polynomials. Curves of degree three must have one double point in order to qualify. 


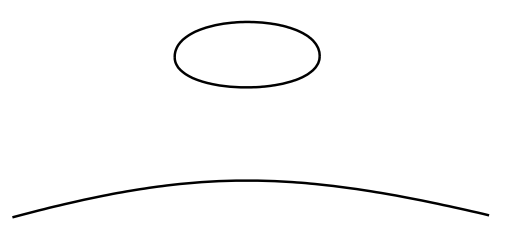

Figure 2. Irreducible Cubic Curve

It is also the case that an irreducible curve of degree $n$ can have at most $(n-1)(n-2) / 2$ double points. Thus, a rational degree $n$ curve has the largest possible number of double points for a curve of its degree. An irreducible curve is one whose implicit equation $f(x, y)=0$ cannot be factored. For example, the degree two curve $x y+x+y+1$ can be factored into $(x+1)(y+1)$ and is thus actually two straight lines. Note that the point at which those two lines intersect is a double point of the curve, even though an irreducible conic cannot have any double points.

Another example of a reducible curve is given by the quartic which is factored into

$$
\left(x^{2}+y^{2}-1\right)\left(x^{2}+y^{2}-4\right)
$$

which is two concentric circles. This leads to another characteristic of rational curves - you can sketch an entire rational curve without removing your pencil from the paper (with the possible exception of a finite number of acnodes). For this reason, the classical algebraic geometry literature sometimes refers to rational curves as "unicursal" curves.

It should also be noted that an algebraic curve which consists of more than one component is not necessarily reducible. For example, many non-rational cubic curves consist of an oval and a branch which does not touch the oval, and yet the equation does not factor.

\section{Detecting Double Points}

How does one verify the existence of a double point? A computational method is to verify that any straight line through the alleged double point hits the curve at least twice at that point. For example, any curve whose equation has no constant or linear terms has a double point at the origin. Consider the cubic algebraic curve:

$x^{3}-2 x y^{2}+x y+3 y^{2}=0$.

To determine how many times a general line through the origin hits this curve, we define such a line parametrically so that the point $t=0$ on the line corresponds to the origin:

$x=a t, \quad y=b t$.

The intersection of the line and the curve yields

$\left(a^{3}-2 a b^{2}\right) t^{3}+\left(a b+3 b^{2}\right) t^{2}=0$. 


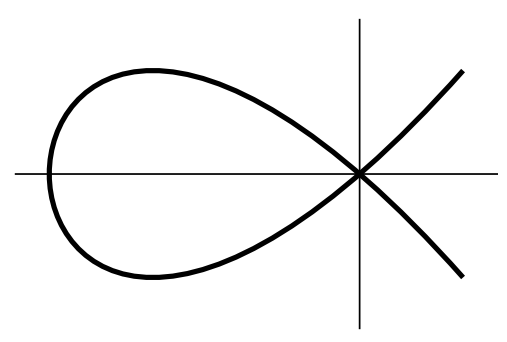

Figure 3. Crunode: $x^{3}+9 x^{2}-12 y^{2}=0$

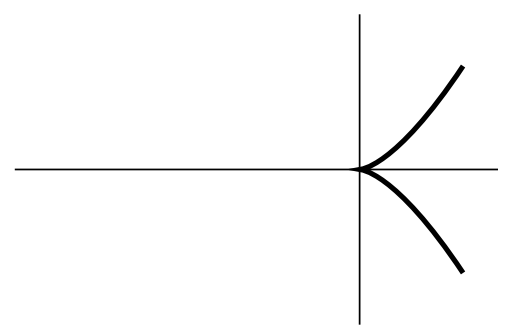

Figure 4. Cusp: $x^{3}-3 y^{2}=0$

Since this equation has at least a double root at $t=0$ for any values of $a$ and $b$ (which control the slope of the line), we conclude that the curve has a double point at the origin.

There are basically three types of double points: the crunode (or simply node, or self intersection), the cusp, and the acnode (or isolated node). An example of a cubic curve with a crunode at the origin is the curve in Figure 9 whose implicit equation is

$x^{3}+9 x^{2}-12 y^{2}=0$.

You can verify by implicitization that this curve can be expressed as a Bézier curve with control points $(3,3),(-13,-15),(-13,15),(3,-3)$.

An example of a cubic curve with a cusp at the origin is given by

$x^{3}-3 y^{2}=0$

which is illustrated in Figure 9.

An example of a curve with an acnode at the origin is shown in Figure 9, given by the equation

$x^{3}-3 x^{2}-3 y^{2}=0$.

We can classify a double point as being a crunode, acnode or cusp by examining the tangent lines to the curve at the double point. A crunode has two distinct real tangent lines, a cusp has two identical tangent lines, and an acnode has two complex tangent 


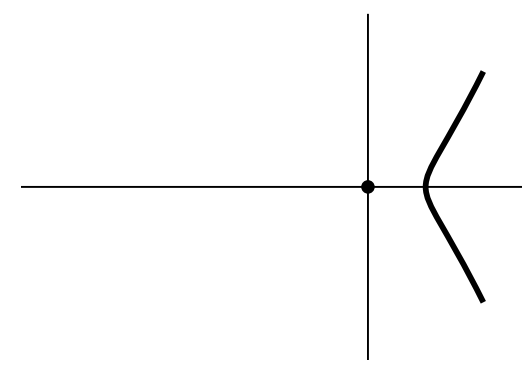

Figure 5. Acnode: $x^{3}-3 x^{2}-3 y^{2}=0$

lines. A tangent line through a double point hits the curve three times at the double point. We can find the slope of the tangent lines by computing values of $a$ and $b$ for which the intersection of the line and the curve has a triple root at $t=0$. In the previous example, this occurs if $a b+3 b^{2}=0$ from which $a=1, b=0$ and $a=-3, b=1$ are the two independent solutions. These represent two real, distinct lines, so the double point in a crunode.

The exercise that we just performed only lets us verify that a given point is singular (a singular point is a double point, triple point, or multiple point in general). To determine the location of singular points, we can use a tool known as the discriminant (discussed later).

\section{Implicit Curve Intersections}

We have seen how the intersections of two Bezier curves can be computed, and also how the intersection points of a parametric and an implicit curve can be found. What about two implicit curves?

One direct method for computing the intersection points of two implicit curves is to take the resultant of the curves with respect to $x$ or $y$. The X-resultant is computed by treating the implicit equations as polynomials in $x$ whose coefficients are polynomials in $y$. The X-resultant eliminates $x$ from the two equations and produces a polynomial in $y$ whose roots are the $y$ coordinates of the intersection points.

We illustrate with a circle $6 x^{2}+6 y^{2}-2 x-15 y-4=0$ and a hyperbola $x^{2}-y^{2}-1=0$.

The x-resultant of these two implicit equations is $144 y^{4}-360 y^{3}+269 y^{2}-60 y$ whose roots are $y=0, y=4 / 3, y=3 / 4$, and $y=5 / 12$. These are the $y$-coordinates of the points of intersection of the two curves.

We can use the y-resultant to find the $\mathrm{x}$-coordinates of the points of intersection. The $\mathrm{y}$-resultant is $144 x^{4}-48 x^{3}-461 x^{2}+40 x+325$ which has roots $x=1, x=5 / 3$, $x=-5 / 4$, and $x=-13 / 12$.

We now find ourselves in the interesting situation of knowing the $x$ and $y$ components of the points of intersection, but we don't know which $x$ goes with which $y$ ! One way to determine that is simply to evaluate each curve equation with every $x$ and every $y$ to 


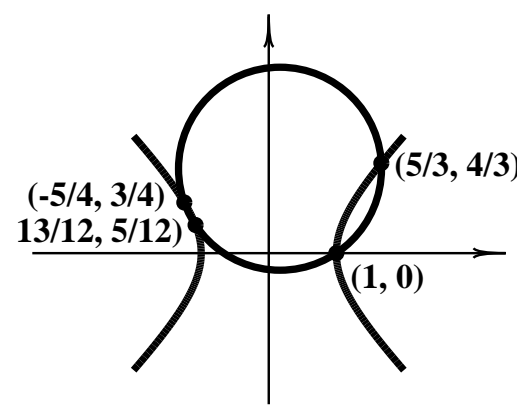

Figure 6. Circle and Hyperbola

see which $(x, y)$ pairs satisfy both curve equations simultaneously. A more clever way is to use Euclid's algorithm. In fact, Euclid's algorithm spares us the trouble of computing both the x-resultant and the y-resultant.

Suppose we had only computed the y-resultant and we wanted to find the y-coordinate of the point of intersection whose $\mathrm{x}$-coordinate is $5 / 3$. That is to say, we want to find a point $\left(\frac{5}{3}, y\right)$ which satisfies both curve equations. We substitute $x=5 / 3$ into the circle equation to get $16 / 6-y^{2}=0$ and into the hyperbola equation to get $6 y^{2}-15 y+28 / 3=0$. We now simply want to find a value of $y$ which satisfies both of these equations. Euclid's algorithm tells us that the GCD of these two $3 y-4=0$, and thus one point of intersection is $\left(\frac{5}{3}, \frac{4}{3}\right)$.

\section{Discriminants}

The discriminant of a univariate polynomial is the resultant of the polynomial and its first derivative. If the discriminant is zero, the polynomial has a double root. (Why?) We all encountered discriminants as early as 7th grade in connection with the quadratic equation. For a degree two polynomial $a t^{2}+b t+c$, the value $b^{2}-4 a c$ is referred to as the discriminant, although if we actually compute the resultant of $a t^{2}+b t+c$ and its derivative $2 a t+b$, we find that the discriminant is actually $a^{2}\left(b^{2}-4 a c\right)$. However, since it is understood that $a \neq 0$, the discriminant can only vanish if $b^{2}-4 a c=0$.

It is also possible to compute the discriminant of an implicit curve by taking the resultant of the implicit equation and its partial derivative with respect to one of the variables. To do this, we treat the implicit equation as a polynomial in $y$ whose coefficients are polynomials in $x$ (or, vice versa). The resultant will then be a polynomial in $x$ with constant coefficients. The roots of that polynomial will correspond to the $x$ coordinates of the vertical tangents and of any double points.

Graphically, the discriminant can be thought of as the silhouette of the curve.

\section{Parameterizing Curves}

There are several ways in which a parametrization may be imposed on a curve of genus zero. For a conic, we may establish a one-one correspondence between points on the 


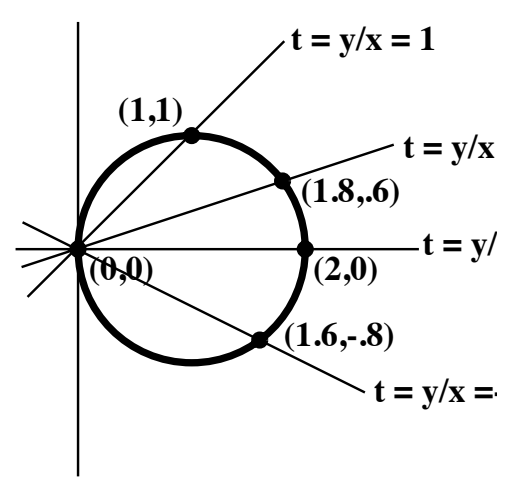

Figure 7. Parametrizing a Circle

curve and a family of lines through a point on the curve. This is most easily illustrated by translating the curve so that it passes through the origin, such as does the curve

$x^{2}-2 x+y^{2}=0$

which is a unit circle centered at $(0,1)$. We next make the substitution $y=t x$ and solve for $x$ as a function of $t$ :

$x^{2}\left(1+t^{2}\right)-2 x=0$

$x=\frac{2}{1+t^{2}}$

$y=t x=\frac{2 t}{1+t^{2}}$

Notice that $y=t x$ is a family of lines through the origin. A one parameter family of lines (or, of any implicitly defined curves) is known as a pencil of lines or curves. The variable line $y=t x$ intersects the curve once at the origin, and at exactly one other point (because of Bezout's theorem). Thus, we have established a one-one correspondence between points on the curve and values $t$ which correspond to lines containing that point and the origin.

An example of a circle parametrized in this manner is shown in Figure 7.

The same trick can be played with a genus zero cubic curve which has been translated so that its double point lies on the origin. if this happens, its implicit equation involves terms of degree two and three only. For example, consider the curve

$\left(x^{3}+2 x^{2} y+3 x y^{2}+4 y^{3}\right)+\left(5 x^{2}+6 x y+7 y^{2}\right)=0$

which has a double point at the origin. Again make the substitution $y=t x$ to obtain $x^{3}\left(1+2 t+3 t^{2}+4 t^{3}\right)+x^{2}\left(5+6 t+7 t^{2}\right)=0$. 


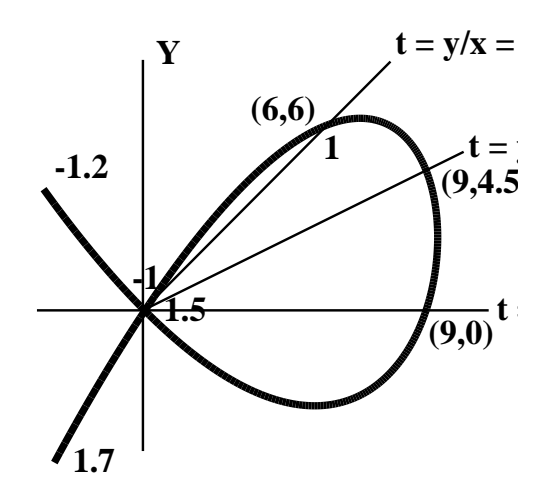

Figure 8. Parametrizing a Cubic Curve

From which

$$
x=-\frac{5+6 t+7 t^{2}}{1+2 t+3 t^{2}+4 t^{3}}, \quad y=t x=-\frac{5 t+6 t^{2}+7 t^{3}}{1+2 t+3 t^{2}+4 t^{3}}
$$

An example of a cubic curve parametrized in this manner is shown in Figure 8.

In general, a cubic curve does not have a double point, and if it does, that double point is not generally at the origin. Thus, before one can parametrize a cubic curve, one must first compute the location of its double point (or, determine that rational parametrization is not possible if there is no double point).

Double points satisfy the equations $f(x, y)=f_{x}(x, y)=f_{y}(x, y)=0$. Consider the cubic curve

$f(x, y)=-21+46 x-13 x^{2}+x^{3}+25 y-23 x y+3 x^{2} y-9 y^{2}+3 x y^{2}+y^{3}$

for which

$f_{x}(x, y)=46-26 x+3 x^{2}-23 y+6 x y+3 y^{2}$

and

$f_{y}(x, y)=25-23 x+3 x^{2}-18 y+6 x y+3 y^{2}$

From section 10, we compute the $x$ coordinates of the intersections of $f_{x}=0$ and $f_{y}=0$ by taking the resultant of $f_{x}$ and $f_{y}$ with respect to $y$ :

$\operatorname{Resultant}\left(\mathrm{f}_{\mathrm{x}}, \mathrm{f}_{\mathrm{y}}, \mathrm{y}\right)=174-159 \mathrm{x}+36 \mathrm{x}^{2}$

whose roots are $x=2$ and $x=\frac{29}{12}$. Likewise the $y$ coordinates of the intersections of $f_{x}=0$ and $f_{y}=0$ are found by taking the resultant of $f_{x}$ and $f_{y}$ with respect to $x$ :

$\operatorname{Resultant}\left(\mathrm{f}_{\mathrm{x}}, \mathrm{f}_{\mathrm{y}}, \mathrm{x}\right)=297-207 \mathrm{y}+36 \mathrm{y}^{2}$

whose roots are $y=3$ and $y=\frac{11}{4}$. From these clues, we find that the only values of $(x, y)$ which satisfy $f(x, y)=f_{x}(x, y)=f_{y}(x, y)=0$ are $(x, y)=(2,3)$, which is therefore the double point. 
This curve can be parametrized by translating the implicit curve so that the double point lies at the origin. This is done by making the substitution $x=x+2, y=y+3$, yielding

$2 x^{2}+x^{3}+7 x y+3 x^{2} y+6 y^{2}+3 x y^{2}+y^{3}$

Parametrization is then performed using the method discussed earlier in this section,

$x=-\frac{6 t^{2}+7 t+2}{t^{3}+3 t^{2}+3 t+1} ; \quad y=-\frac{6 t^{3}+7 t^{2}+2 t}{t^{3}+3 t^{2}+3 t+1}$

and the parametrized curve is translated back so that the doubled point is again at $(2,3)$ :

$x=-\frac{6 t^{2}+7 t+2}{t^{3}+3 t^{2}+3 t+1}+2=\frac{2 t^{3}-t}{t^{3}+3 t^{2}+3 t+1} ;$

$y=-\frac{6 t^{3}+7 t^{2}+2 t}{t^{3}+3 t^{2}+3 t+1}+3=\frac{-3 t^{3}+2 t^{2}+7 t+3}{t^{3}+3 t^{2}+3 t+1}$

\section{REFERENCES}

1. Abhyankar,S. and Bajaj,C. (1987), Automatic parameterization of rational curves and surfaces I: Conics and conicoids, Computer Aided Design 19(1): 11-14.

2. Abhyankar,S. and Bajaj,C. (1987), Automatic parameterization of rational curves and surfaces II: Cubics and cubicoids, Computer Aided Design 19(9): 499-502.

3. Abhyankar,S. and Bajaj,C. (1988), Automatic parameterization of rational curves and surfaces III: Algebraic plane curves, Computer Aided Geometric Design 5: 309-321.

4. Abhyankar,S. and Bajaj,C. (1989),Automatic parameterization of rational curves and surfaces IV: Algebraic space curves, ACM Transactions on Graphics 8(4): 324-333.

5. Abhyankar, S.S. (1990), Algebraic Geometry for Scientists and Engineers, American Mathematical Society, Providence, R.I., 1990.

6. Adams, W. and Loustaunau, P. (1994), An introduction to Gröbner bases, American Mathematical Society, Providence, R.I., 1994.

7. Alonso,C., Gutierrez,J., and Recio,T. (1995), An implicitization algorithm with fewer variables, Computer Aided Geometric Design 12(3): 251-258.

8. Bajaj,C. and Ihm,I. (1992), Smoothing of polyhedra with implicit algebraic splines, Computer Graphics 26(2): 79-88, SIGGRAPH 92, Chicago, Illinois,

9. Bajaj,C., Chen,J. and Xu,G. (1995), Modeling with Cubic A-patches, ACM Transactions on Graphics 14(2): 103-133.

10. Bajaj,C. (1997), Implicit surface patches, Introduction to Implicit Surfaces, edited by J. Bloomenthal, Morgan Kaufman Publishers, 98-125.

11. Bajaj,C., Holt,R., and Netravali,A. (1998), Rational parameterizations of nonsingular cubic surfaces, ACM Transactions on Graphics 17(1): 1 - 31.

12. Bajaj,C. and Xu,G. (1999), A-splines: Local interpolation and approximation using $G^{k}$ - continuous piecewise real algebraic curves, Computer Aided Geometric Design 16.

13. Becker, T. and Weispfenning, V. (1993), Gröbner Bases: A computational approach to commutative algebra, Springer-Verlag. 
14. Boehm,W. and Prautzsch,H. (1993), Geometric Concepts for Geometric Design, A K Peters, Welleley, Massachusetts.

15. Buchberger,B. (1965), Ein Algorithmus zum Auffinden der Basiselemente des Restklassenringes Nach Einem Nulldimensionalen Polynomideal, Ph.D. Thesis (in German), Universitat Innsbruck, Austria.

16. Buchberger,B. (1985), Gröbner bases: an algorithmic method in polynomial ideal theory, Multidimensional Systems Theory, N.K.Bose, ed., D.Reidel Publishing Co., Netherlands, pp.184-232.

17. Chionh,E. (1990), Base Points, Resultants, and the Implicit Representation of Rational Surfaces, Ph.D. Thesis, Dept. of Computer Science, University of Waterloo, Canada.

18. Chionh,E. and Goldman,R. (1992), Using multivariate resultants to find the implicit equation of a rational surface, The Visual Computer 8: 171-180.

19. Chionh,E. and Goldman,R. (1992), Degree, multiplicity, and inversion formulas for rational surfaces using u-resultants, Computer Aided Geometric Design 9: 93-108.

20. Chionh,E. and Goldman,R. (1995), Elimination and Resultants, Part 1: Elimination \& Bivariate Resultants, IEEE Computer Graphics and Applications 15(1): 69-77.

21. Chionh,E. and Goldman,R. (1995), Elimination and Resultants, Part 2: Multivariate Resultants, IEEE Computer Graphics and Applications 15(2): 60-69.

22. Cox,D., Little,J., and O'Shea,D. (1992), Ideals, Varieties and Algorithms, SpringerVerlag.

23. Cox,D., Little,J., and O'Shea,D. (1998), Using Algebraic Geometry, Springer-Verlag.

24. Cox,D., Sederberg,T., and Chen,F. (1998), The moving line ideal basis of planar rational curves, Computer Aided Geometric Design 15:803-827.

25. Cox,D., Zhang,M., and Goldman,R. (2000), On the validity of implicitization by moving quadrics for rational surfaces with no base points, Journal of Symbolic Computation 29: 419-440.

26. De Montaudouin,Y. and Tiller,W. (1984), The Cayley method in computer aided geometric design, Computer Aided Geometric Design 1:309-326.

27. Dixon,A.L. (1908), The eliminant of three quantics in two independent variables, Proc. London Math. Soc. 6(Ser.2): 468-478.

28. Gao,X. and Chou,S. (1992), Implicitization of rational parametric equations, Journal of Symbolic Computation 14: 459-470.

29. Goldman,R., Sederberg,T., and Anderson,D. (1984), Vector elimination: A technique for the implicitization, inversion, and intersection of planar parametric rational polynomial curves, Computer Aided Geometric Design 1:327-356.

30. Goldman,R. (1985), The method of resolvents: A technique for the implicitization, inversion, and intersection of non-planar, parametric, rational cubic curves, Computer Aided Geometric Design 2(4): 237-255.

31. Goldman,R. and Sederberg,T. (1985), Some applications of resultants to problems in computational geometry, The Visual Computer 1(2): 101-107.

32. Gonzalez-Vega,L. (1997), Implicitization of parametric curves and surfaces by using multidimensional Newton formulae, Journal of Symbolic Computation 23(2/3): 137151.

33. Hoffmann,C. (1989), Geometric and Solid Modeling, an Introduction, Morgan Kauf- 
mann, San Mateo.

34. Hoffmann,C. (1993), Implicit curves and surfaces in computer-aided geometric design, IEEE Computer Graphics and Applications 13(1): 79-88.

35. Jacobsen,N. (1974), Basic Algebraic I, Freeman, San Francisco.

36. Jacobsen,N. (1980), Basic Algebraic II, Freeman, San Francisco.

37. Kapur,D. (1992),Elimination methods: an introduction, In: Symbolic and Numerical Computation for Artificial Intelligence, eds. By Donald,B. and Kapur,D. and Mundy,J., pp.45-87, Academic Press.

38. Manocha,D. and Canny,J. (1992), Implicit representation of rational parametric surfaces, Journal of Symbolic Computation 13(5): 485-510.

39. Manocha,D. and Canny,J. (1992), Algorithm for implicitizing rational parametric surfaces, Computer Aided Geometric Design 9(1): 25-51.

40. Salmon,G. (1866), Modern Higher Algebra, G.E.Steckert \& Co., New York, 1985.

41. Salmon,G. (1914), A Treatise on the Analytic Geometry of Three Dimensions, Vol I, II, Ed., R.Rogers, Reprintd, Chelsea Publishing

42. Sederberg,T. (1983), Implicit and Parametric Curves and Surfaces, Ph.D. Thesis, Purdue University, West Lafayette,IN.

43. Sederberg,T., Anderson,D., and Goldman,R. (1984), Implicit representation of parametric curves and surfaces, Computer Vision, Graphics and Image Processing 28: $72-84$.

44. Sederberg,T. and Goldman,R. (1986), Algebraic geometry for computer-aided geometric design, IEEE Computer Graphics and Applications 6(6):5-59.

45. Sederberg,T. (1987), Algebraic geometry for surface and solid modeling, In Geometric Modeling: Algorithms and Trends, G.Farin ed., Philadelphia, SIAM Publication.

46. Sederberg,T. and Snively,J. (1987), Parameterizing cubic algebraic surfaces, in The Mathematics of Surfaces II, R. R. Martin, ed., Oxford University Press, Oxford UK, pp. 299-320.

47. Sederberg,T., Saito,T., Qi,D., and Klimaszewski,K. (1994), Curve implicitization using moving lines, Computer Aided Geometric Design 11: 687-706.

48. Sederberg,T. and Chen,F. (1995), Implicitization using moving curves and surfaces, SIGGRAPH 95, Computer Graphics Proceedings, Annual Conference Series, pp.301308.

49. Sederberg,T., Goldman,R., and Du,H. (1997), Implicitizing rational curves by the method of moving algebraic curves, Journal of Symbolic Computation 23:153-175.

50. Sederberg,T. (1998), Applications to computer aided geometric design, Proceedings of Symposia in Applied Mathematics, Volume 53, pp.67-89.

51. Sederberg,T., Zheng,J., Klimaszewski,K. and Dokken,T. (1999), Approximate implicitization using monoid curves and surfaces, Graphical Models and Image Processing 61(4): 177-198.

52. Semple,J. and Roth,L., (1949), Introduction to Algebraic Geometry, Oxford University Press, Oxford, U.K.

53. Sendra,J. and Winkler,F. (1991), Symbolic parametrization of curves, Journal of Symbolic Computation 6: 607-632 .

54. Sendra,J. and Winkler,F. (1998), Real parametrization of algebraic curves, Artificial intelligence and symbolic computation (Plattsburgh, NY), 284-295. 
55. Van der Waerden,B.L. (1950), Modern Algebra, Frederick Ungar, New York, 2nd edition

56. Walker,R. (1949), Algebraic Curves, Dover Publications, New York.

57. Warren,J. (1989), Blending algebraic surfaces, ACM Transactions on Graphics 8:263278.

58. Wu,W.-T. (1986), Basic principles of mechanical theorem proving in geometries, J. Systems Sciences and Mathematical Sciences 4:207-235.

59. Zhang,M., Chionh,E., and Goldman,R. (1999), On a relationship between the moving line and moving conic coefficient matrices, Computer Aided Geometric Design 16:517527. 